\title{
Initiatives for acausal model connection using FMI in JSAE (Society of Automotive Engineers of Japan)
}

\author{
Yutaka Hirano $^{1}$ Satoshi Shimada ${ }^{2} \quad$ Yoichi Teraoka $^{3} \quad$ Osamu Seya $^{4}$ \\ Yuji Ohsumi $^{5} \quad$ Shintaroh Murakami $^{6}$ Tomohide Hirono $^{7}$ Takayuki Sekisue ${ }^{8}$ \\ ${ }^{1}$ Toyota Motor Corporation, Japan, yutaka hirano@mail. toyota.co.jp \\ ${ }^{2}$ Honda R\&D Co., Ltd., Japan, satoshi_shimada@n.t.rd.honda.co.jp \\ ${ }^{3}$ Mazda Motor Corporation, Japan, teraoka. yo@mazda.co.jp \\ ${ }^{4}$ DENSO CORPORATION, Japan, OSAMU SEYA@denso.co.jp \\ ${ }^{5}$ AZAPA Co., Ltd., Japan, yuji-oosumi@azapa.co.jp \\ ${ }^{6}$ Dassault Systèmes K.K., Japan, Shintaroh. MURAKAMI @ 3ds . com \\ ${ }^{7}$ NewtonWorks Corporation, Japan, hirono.tomohi de@ newtonworks. co.jp \\ ${ }^{8}$ ANSYS Japan K.K., Japan, takayuki.sekisuedansys . com
}

\begin{abstract}
Authors initiated trial and evaluation of a new method to connect physical ports of acausal model and causal signal ports using FMI as an activity of technical committee of JSAE (Society of Automotive Engineers of Japan). We propose a way of model export and connection using new adaptor models. This method was tested by a benchmark model of a control system. Simulation results for the benchmark model showed good consistency between the original acausal model and the connected model using FMUs separated from the original model by this method. Also a guide-line about using FMI for the model connection using this method was made in JSAE and is distributed to general users of Japanese automotive industries. Finally expectations about future enhancement of FMI for model exchange and circulation between different companies are presented.
\end{abstract}

Keywords: FMI, Model Exchange, Acausal Physical Connector

\section{Introduction}

Importance of utilizing simulation is increasing for the development of automotive systems because both high functionality and high reliability are required while the development time is becoming shorter. For large-scale and multi-domain development of automotive systems, environment for development which enables to connect simulation models developed in various companies is becoming important more and more. Though, in automotive industries in Japan, various kinds of physical modeling tools are used for each physical domain by each organization. Thus it was difficult to connect those models easily.

Upon above background, the Committee on Research of Model Development and Circulation Methods Based on Global Standardized Description was established since March 2012 in JSAE. In the
Committee, Working Group for Model Connection Technologies was started to try and evaluate the efficacy of model connection using FMI. In that activity authors found that there was a problem of algebraic loop generated by diving and connecting submodels (FMUs) because FMI only provided the way to connect models by causal signal flows, i.e. defining causality of input and output of components is required. On the other hand, acausal modeling tools as Modelica tools can handle the problem of algebraic loop by causality analysis and symbolic manipulation of equations. Thus it seemed effective to connect FMUs in acausal modeling environment by converting causal signal ports to acasual physical port and vice versa. In the following section, the method to use special adaptor models to divide and connect sub-models via FMI is proposed. The method was validated by benchmark models developed in JSAE committee. Comparison between the simulation result of the original acausal model and that of a unified model of divided FMUs was done and we got good consistency of the simulation results. Finally some expectations for future enhancement of FMI for model exchange from Japanese automotive industries are described.

\section{Benchmark Model of a Simple Control System}

\subsection{Structure of the Benchmark Model}

Figure 1 shows one benchmark model of JSAE used for the evaluation of model connection methods using FMI. This model is a simple control system of DC motor for rotational angle feedback control as shown in Figure 2.

This simple control system consists of three major sub-systems: PI controller, DC motor and rotational mechanical system. In each sub-system, the system of equations are as follows. 


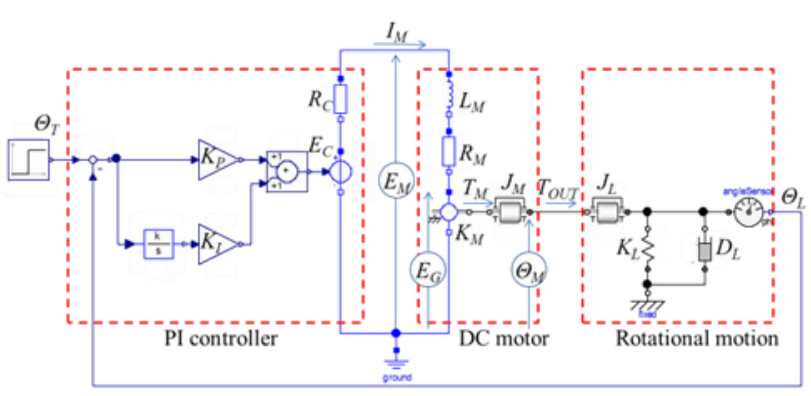

Figure 1. Benchmark model (Simple control system)

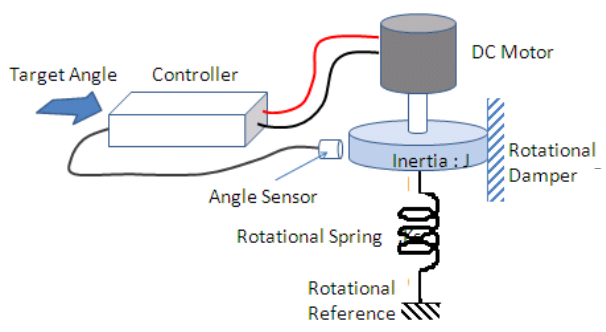

Figure 2. Physical image of the simple control system

PI Controller:

$$
\begin{gathered}
E_{c}(s)=K_{P} \cdot\left[\Theta_{T}(s)-\Theta_{L}(s)\right]+K_{I} \cdot \frac{1}{s}\left[\Theta_{T}(s)-\Theta_{L}(s)\right] \\
E_{M}(s)=E_{C}(s)-R_{C} \cdot I_{M}(s)
\end{gathered}
$$

DC Motor:

$$
\begin{gathered}
E_{M}(s)=L_{M} \cdot s I_{M}(s)+R_{M} \cdot I_{M}(s)+E_{G}(s) \\
E_{G}(s)=K_{M} \cdot s \Theta_{M}(s) \\
T_{M}(s)=K_{M} \cdot I_{M}(s) \\
T_{\text {OUT }}(s)=T_{M}(s)-J_{M} \cdot s^{2} \Theta_{M}(s)
\end{gathered}
$$

Rotational Mechanical System:

$$
\begin{gathered}
T_{\text {OUT }}(s)=J_{L} \cdot s^{2} \Theta_{L}(s)+D_{L} \cdot s \Theta_{L}(s)+K_{L} \cdot \Theta_{L}(s) \\
\Theta_{M}(s)=\Theta_{L}(s)
\end{gathered}
$$

Here,

$E_{c}(s)$ : Voltage of voltage generator in PI controller

$E_{M}(s)$ : Terminal voltage of DC motor

$E_{G}(s)$ : Electromotive voltage of DC motor

$I_{M}(s)$ : Current flow of DC motor

$T_{M}(s)$ : Driving torque of DC motor

$T_{\text {OUT }}(s)$ : Output torque of DC motor

$\Theta_{T}(s)$ : Target rotational angle

$\Theta_{M}(s)$ : Rotational angle of DC motor

$\Theta_{L}(s)$ : Rotational angle of the mechanical system and

$K_{P}$ : Proportional gain of PI controller

$K_{I}$ : Integration gain of PI controller

$R_{C}$ : Internal resistance of PI controller

$L_{M}$ : Inductance of DC motor

$R_{M}$ : Resistance of DC motor

$K_{M}$ : Current - torque coefficient of DC motor

$J_{M}$ : Inertia of DC motor

$J_{L}:$ Inertia of the rotational mechanical system

$D_{L}$ : Damping coefficient of the mechanical system

$K_{L}$ : Spring coefficient of the mechanical system

\subsection{Adapter Model}

Let's consider to make FMU from the DC motor submodel of Figure 1. Here, one portion to divide the model is the electronic connector between the electronic output of PI controller and input of the DC motor. Also the mechanical connector between the mechanical output of DC motor and the mechanical input of the rotational motion system should be chosen as dividing portion. It is important to choose the appropriate connectors which coincide with the actual interconnection of parts and systems to handle models provided by different suppliers easily.

In general, it is the most convenient that such models are described by acausal modeling tool such as Modelica tools because there is no necessity to consider the causality of each system of equations when assembling the models. But it is still not realized that every model of necessary sub-systems are made by acausal modeling tools. There still are many existing models made by different tools supporting only causal modeling.

Thus, it is important to enable those causal models to be connected by using FMI in acausal modeling environment. However, because FMI is based on only causal signal connection, it is necessary to prepare adaptor models to translate acausal physical port to causal signal connectors. Figure 3 shows the proposed models of adaptors for electric, rotational mechanical and translational mechanical domains.

In electronics adopters shown in Figure 3, the connectors shown by rectangular terminal are acausal physical ports. The connectors shown by triangle terminal are causal signal connectors of voltage and current, and the direction of the triangle head shows the direction of the corresponding signal flow. There are following equations between each variable.

$$
\begin{gathered}
E 1_{\text {pin }}=v 1 \\
i 1=I 1_{\text {pin }} \\
v 2=E 2_{\text {pin }} \\
I 2_{\text {pin }}=-i 2
\end{gathered}
$$

In rotational mechanical adopters shown in Figure 3, the connectors shown by circle terminal are acausal physical flanges. The connectors shown by triangle terminal are causal connectors expressing physical signals of rotational angle, velocity, acceleration and torque. As same as the electronic adaptors, the direction of the triangle head shows the direction of the corresponding signal flow. There are following equations between each variable.

$$
\begin{aligned}
& \Theta 1_{\text {flange }}=\theta 1 \\
& \Omega 1_{\text {flange }}=\omega 1 \\
& A 1_{\text {flange }}=a 1 \\
& \tau 1=T 1_{\text {flange }}
\end{aligned}
$$




\begin{tabular}{|c|c|c|}
\hline Electric & Rotational Mechanical & Translational Mechanical \\
\hline$\prod_{E_{\text {pin }}, I_{\text {pin }}}$ & $\begin{array}{l}\text { Torque signal output } \\
\varphi_{\text {flange }}=p h i \\
\omega_{\text {flange }}=w \\
a_{\text {flange }}=a \\
\tau_{\text {flange }}=\text { tau } \\
\varphi_{\text {flange }}, \omega_{\text {flange }}, a_{\text {flange }}, \tau_{\text {flange }}\end{array}$ & $\begin{array}{l}\text { Force signal output } \\
s_{\text {trfln }}=s \\
v_{\text {trfln }}=v \\
a_{\text {trfln }}=a \\
f_{\text {trfln }}=f \\
s_{\text {trfln }}, v_{\text {trfln }}, a_{\text {trfln }}, f_{\text {trfln }}\end{array}$ \\
\hline $\begin{array}{l}E_{\text {pin }}=v \\
I_{\text {pin }}=-i\end{array}$ & $\begin{array}{l}\text { Angle signal output } \\
\qquad \begin{array}{l}\text { phi }>\varphi_{\text {flange }}=p h i \\
a_{\text {flange }}=w \\
\tau_{\text {flange }}=a \\
\text { flange }\end{array}=- \text { tau } \\
\varphi_{\text {flange }}, \omega_{\text {flange }}, a_{\text {flange }}, \tau_{\text {flange }}\end{array}$ & $\begin{array}{l}\text { Position signal output } \\
s_{\text {trfln }}=s \\
v_{\text {trfln }}=v \\
a_{t r f l n}, v_{t r f l n}, a_{t r f l n}, f_{t r f l n}\end{array}$ \\
\hline
\end{tabular}

Figure 3. Adapter models to connect acausal physical port and causal signal ports

$$
\begin{gathered}
\theta 2=\Theta 2_{\text {flange }} \\
\omega 2=\Omega 2_{\text {flange }} \\
a 2=A 2_{\text {flange }} \\
T 2_{\text {flange }}=-\tau 2
\end{gathered}
$$

There is similar relationship between the signals of acausal physical port and causal signal connectors also for the mechanical translational adaptors.

It is important to notice that there are minus signs in the equation (12) and the equation (20). To decide the sign of every flow variables of acausal connector by integrated way, we define the polarity of flow variables as follows.

- Sign of flow variables defined as output of causal connector is positive when the flow goes out from the component.

- Sign of flow variables defined as input of causal connector is positive when the flow comes into the component.

By above definition, the function of the adaptor models should become like bellows.

- When transferring flow variable(s) coming into the component at acausal connector to that of causal connector, the sign of the variable(s) is plus. (It's not necessary to change the polarity of both signals as shown in the equation (10) and (16).)

- When transferring flow variable(s) going out of the component at causal connector to that of acausal connector, the sign of the variable(s) is minus because of the definition of Modelica Standard Libraries (MSL): i.e. the sign of flow variables is plus when they come into the component. This means that it is necessary to invert the sign of the corresponding flow variables as shown in the equation (12) and (20).

By above definition of signal flows, it becomes possible to utilize the functionality of Modelica tools to generate the equation about flow variables to be summed to zero when the physical connectors are connected. This feature enables us to connect FMUs generated by using proposed adaptors into acausal modeling environment. By this way, it becomes possible to utilize the functionality of Modelica translator to handle algebraic loops for connected model of multiple FMUs. We have proposed the above definition of the adaptor models to Modelica Association in March 2014. It is desired that this kind of adaptor models will be prepared in coming future, hopefully as a part of MSL, also for other physical domains such as heat transition system, liquid system and so on.

\subsection{Generation of FMU}

Next we will show the way to split the sub-model of DC motor by using adaptor models mentioned in the above section. We can make the sub-model as shown in Figure 4 by using one mechanical adaptor and two electronic adaptors.

Equations for the DC motor become as follows.

$$
\begin{gathered}
i_{M 2_{-} \text {OUT }}(s)=\frac{v_{M 1_{-} I N}(s)-v_{M 2_{I N}}(s)-E_{G}(s)}{L_{M} \cdot \mathrm{s}+R_{M}} \\
i_{M 1_{-} \text {OUT }}(s)=-i_{M 2_{-} \text {OUT }}(s) \\
E_{G}(s)=K_{M} \cdot \omega_{M_{-} I N}(s) \\
T_{M}(s)=K_{M} \cdot i_{M 2_{-} \text {OUT }}(s) \\
\tau_{M_{-} \text {OUT }}(s)=T_{M}(s)-J_{M} \cdot a_{M_{-} I N}(s)
\end{gathered}
$$




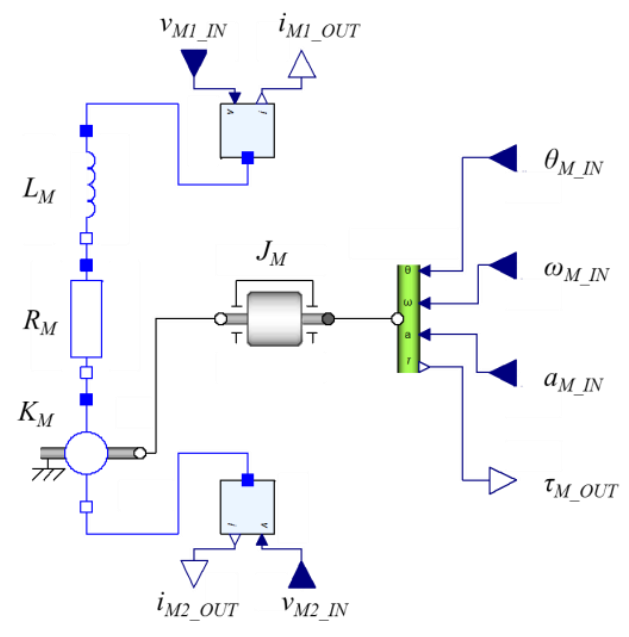

Figure 4. DC motor model with adaptors

Now we can generate FMU from the sub-model of the DC motor shown in Figure 4 because all of the variables which are used for interconnecting to other models are defined by causal (i.e. defined as either of input or output) connectors.

\subsection{Importing and Connection of generated FMU}

When importing the generated FMU in the host tool (i.e. by Import for ModelExchange or Master for Cosimulation), the imported FMU looks like Figure 5. (The appearance is different according to the used tool.)

Inside the imported FMU model, the relationship between the input and output becomes as following equations (26) to (28).

$$
\begin{gathered}
i_{M 2_{\text {OUT }}} \\
=f_{1}\left(v_{M 1_{-} I N}, v_{M 2_{-} I N}, \theta_{M_{-} I N}, \omega_{M_{-} I N}, a_{M_{-} I N}\right) \\
i_{M 1_{-} \text {OUT }}=-i_{M 2_{-} O U T} \\
\tau_{M_{-} \text {OUT }}=f_{2}\left(v_{M 1_{-} I N}, v_{M 2_{-} I N}, \theta_{M_{-} I N}, \omega_{M_{-} I N}, a_{M_{-} I N}\right)
\end{gathered}
$$

The definition of each function $f_{1}$ and $f_{2}$ cannot be seen from outside. The actual equation of functions are implemented as dll file in the fmu file (in the case of using Windows OS).

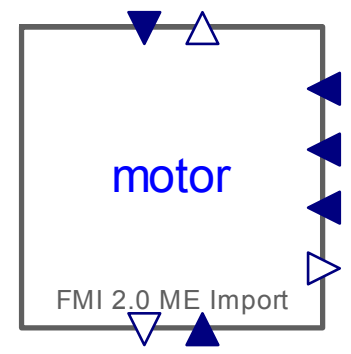

Figure 5. Imported FMU model of DC motor

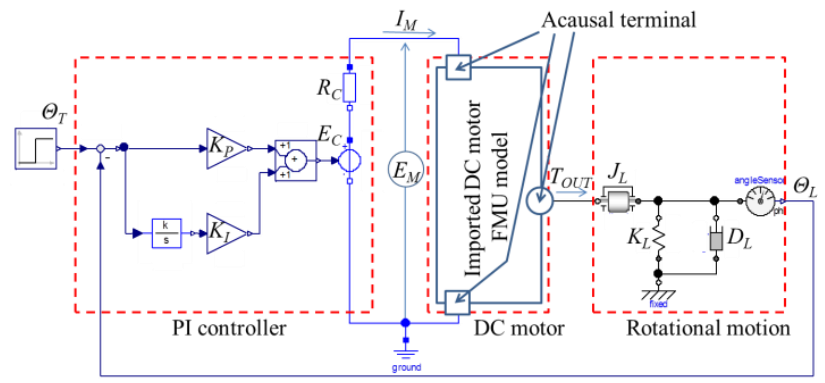

Figure 6. System model connecting imported DC motor FMU model

Next, we will connect the imported FMU model of the DC motor within the total system model as shown in Figure 6. In this model, the acausal sub-model of DC motor in Figure 1 is just replaced by the imported FMU model. The problem is how to connect the causal FMU model to the acausal physical model of the total system. We can solve this problem also by using adaptor models mentioned above. In the case that the FMU model outputs voltage signal and inputs current signal, then we should connect the adaptor which connects electronic acausal port with voltage signal as input and current signal as output shown in Figure 3. Similarly, if a ternimal of rotational mechanics outputs torque signal and inputs signals of rotational angle, velocity and acceleration, then we should connect the adaptor which connects mechanical acausal connector with torque signal as input and signals of rotational angle, velocity and acceleration as output shown in Figure 3. In the case of FMU model shown in Figure 5, the necessary model connected with adaptor models becomes like Figure 7.

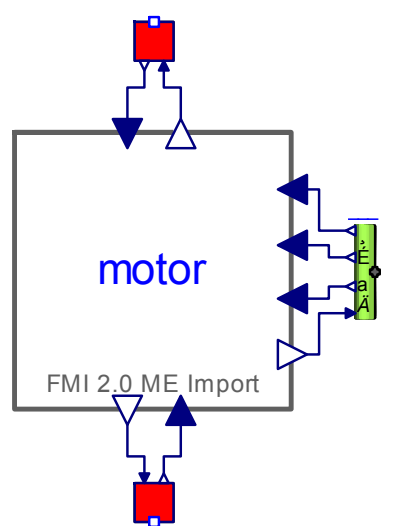

Figure 7. FMU model with adaptors

Now we can connect the FMU model with adaptors into the acasaul total model as shown in Figure 6. Figure 8 shows the result of connecting the FMU model of the DC motor using the adaptors. 


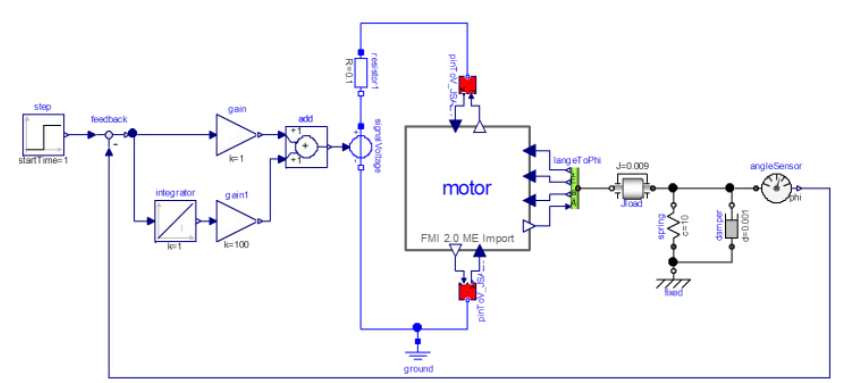

Figure 8. System model with DC motor FMU model

Similarly, separated FMU models of PI controller and rotational motion system can be generated by using proper adaptor models as shown in Figure 9. Then generated and imported FMU models of those subsystems become as shown in Figure 10. Finally we can make a total system model using those three FMUs as shown in Figure 11. In this example, FMU of both PI controller and DC motor output current signal. Also FMU of both DC motor and rotational motion system output torque signal. Please be aware that by using the adaptors it becomes possible to connect output signals each other between two FMUs via acausal connection because causality is solved by Modelica translator. It becomes error if the causal output signals of FMU are directly connected without using adaptors.

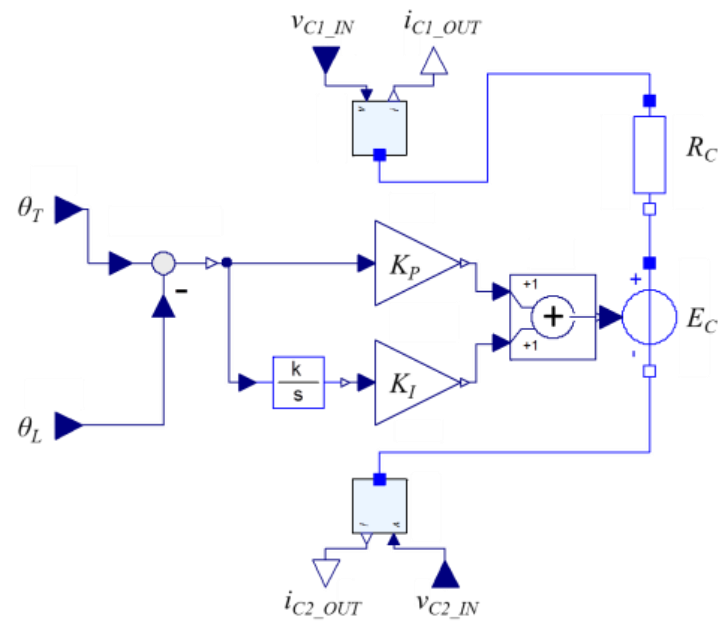

(a) PI controller

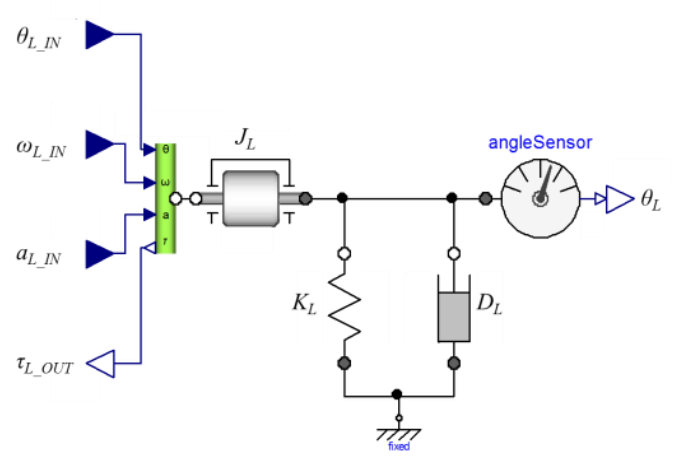

(b) Rotational motion system
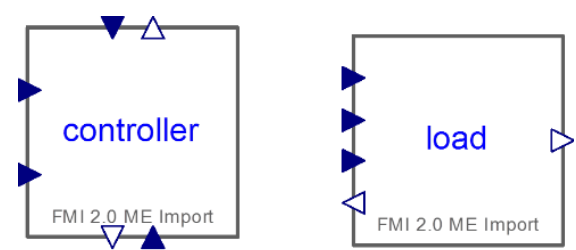

(a) PI controller (b) Rotational motion system

Figure 10. Imported FMU models

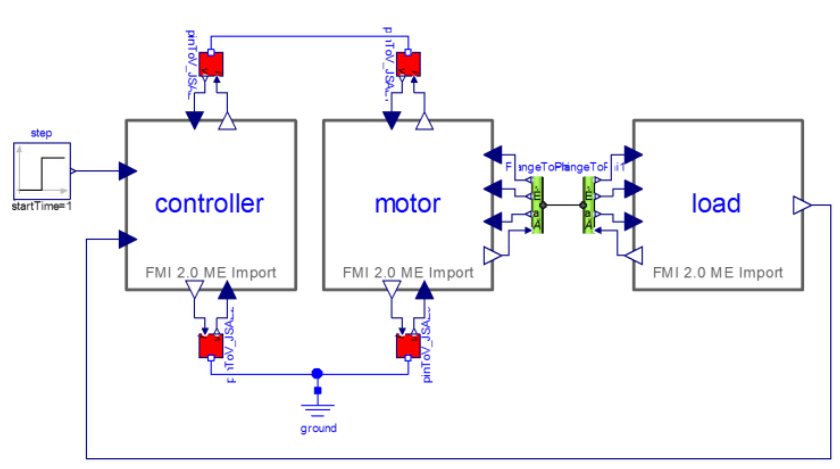

Figure 11. System model using 3 FMUs

\subsection{Simulation Results}

Figure 12 shows simulation results of the rotational angle of load shaft for the benchmark model. The results of the original model without using FMU (Figure 1), the model using only FMU of DC motor (Figure 8) and the model using all three FMUs (Figure 11) are compared. It is confirmed that all the results are identical. Here, the parameter of the models are set so that the results become oscillatory and the comparison is easy. All FMUs were made by ModelExchange mode.

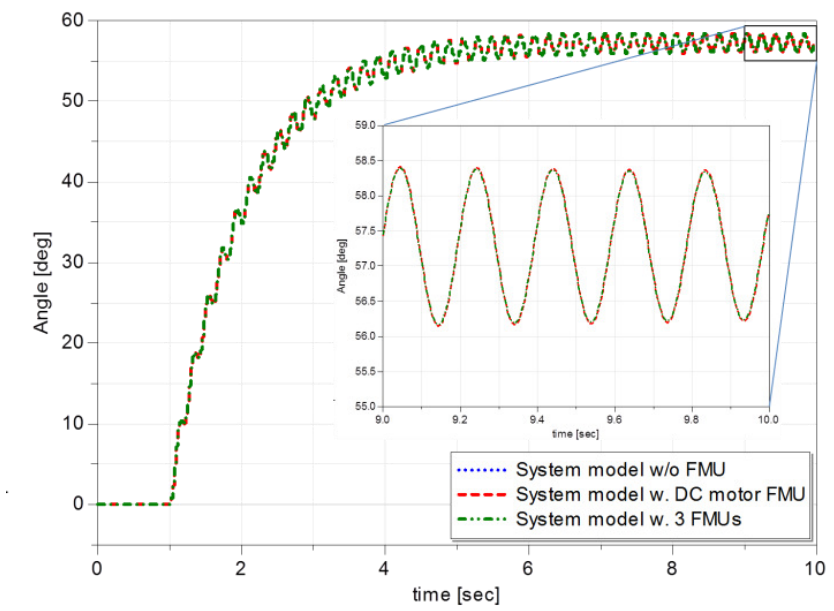

Figure 12. Simulation results (Rotation angle of load shaft)

Figure 9. Separated models for FMU 


\section{Full Vehicle Simulation Using FMUs from Different Tools}

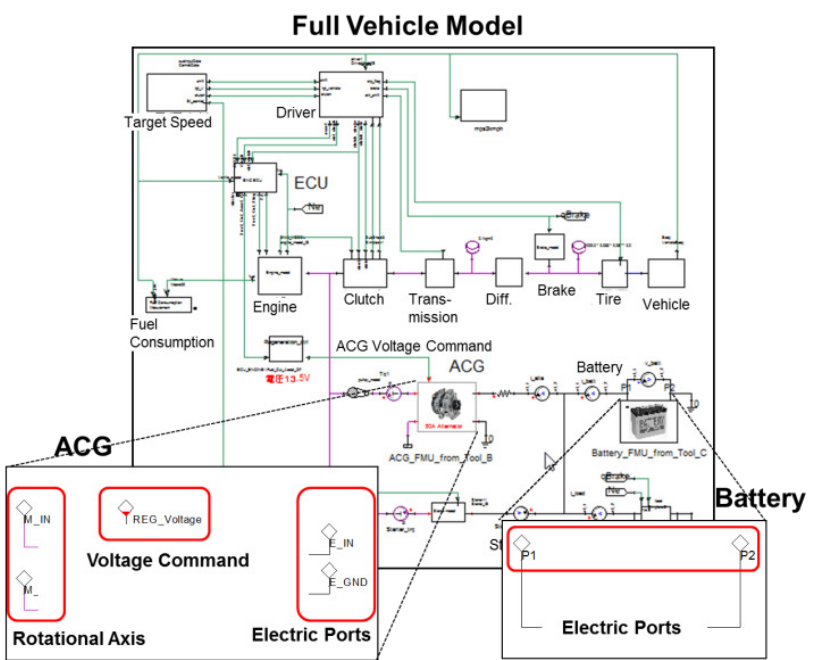

Figure 13. Full vehicle model for electric system evaluation

Another test model to evaluate the electric system using full vehicle model was developed and tested. Figure 13 shows the total model of the test model generated by one VHDL-AMS tool (Sekisue et al., 2013). Here, we tried to replace sub-models of AC generator (ACG) and battery with FMUs generated by other Modelica tools. As same as the benchmark model shown in Figure 1, we introduced adaptor models to connect acausal physical terminals and signals of causal connectors.

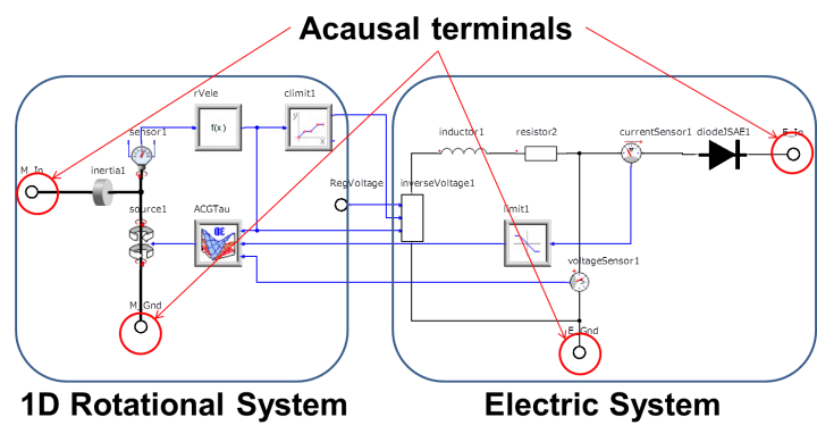

Figure 14. Model of ACG

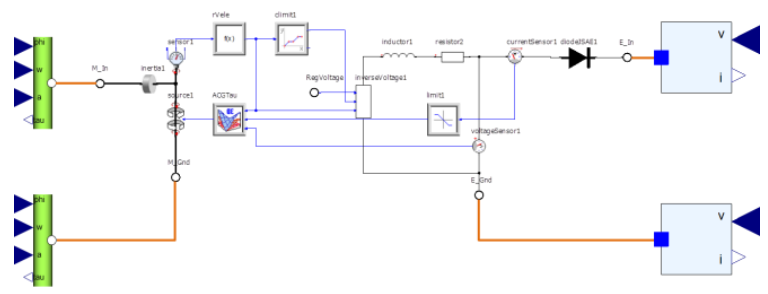

Figure 15. Model of ACG with adaptor models

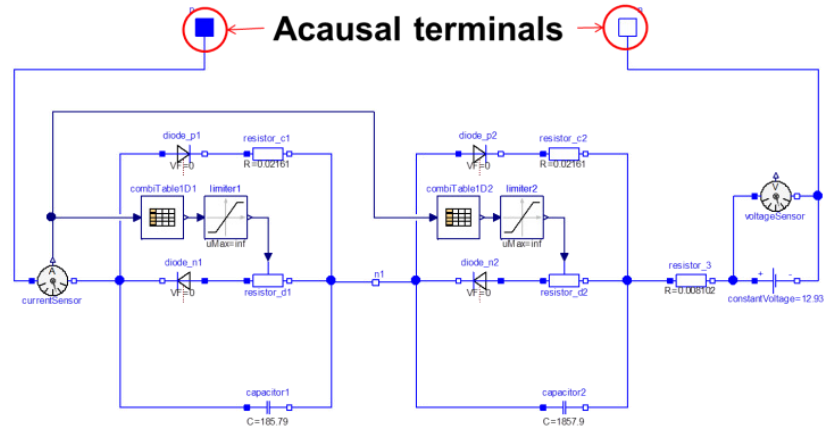

Figure 16. Model of battery

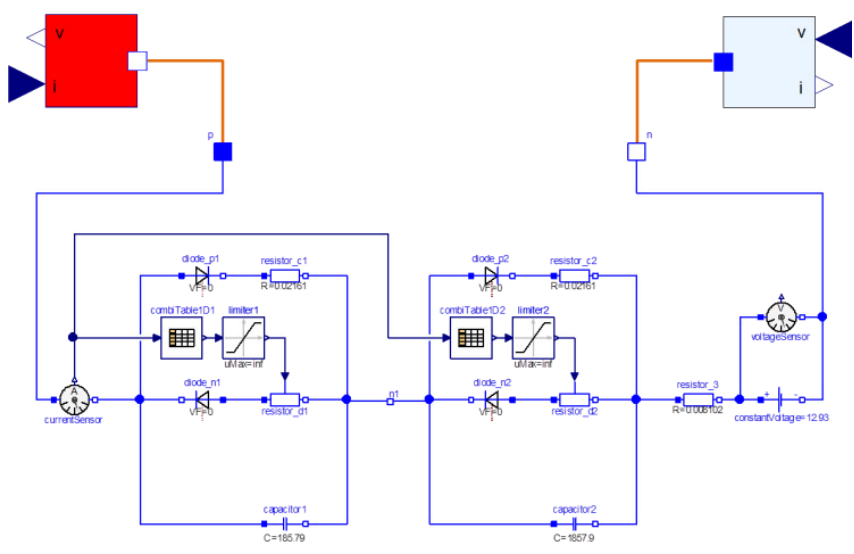

Figure 17. Model of battery with adaptor models

Figure 14 shows the model of ACG generated by one Modelica tool. The model to be converted to FMU was generated as shown in Figure 15 by adding the adaptor models. On the other hand, battery model was made using another Modelica tool as shown in Figure 16. Similarly, the modified model to be converted to FMU was made as shown in Figure 17. It was necessary to make the above-mentioned adaptor models also in the VHDL-AMS tool and connect FMUs from each Modelica tool as shown in Figure 18.

Finally simulation test was done in the VHDL-AMS tool. One example of the results is shown in Figure 19. It was confirmed that model connection using FMUs from different tools was successful by using the proposed adaptor models.

\section{Summary and Future Requests}

We proposed and tested a method using some kind of adaptor models for FMU to realize following functions.

1. Generating FMUs which have connectors of causal signals from acausal modeling tool.

2. Converting causal FMUs to acausal sub-models and connecting them by acausal modeling way. 


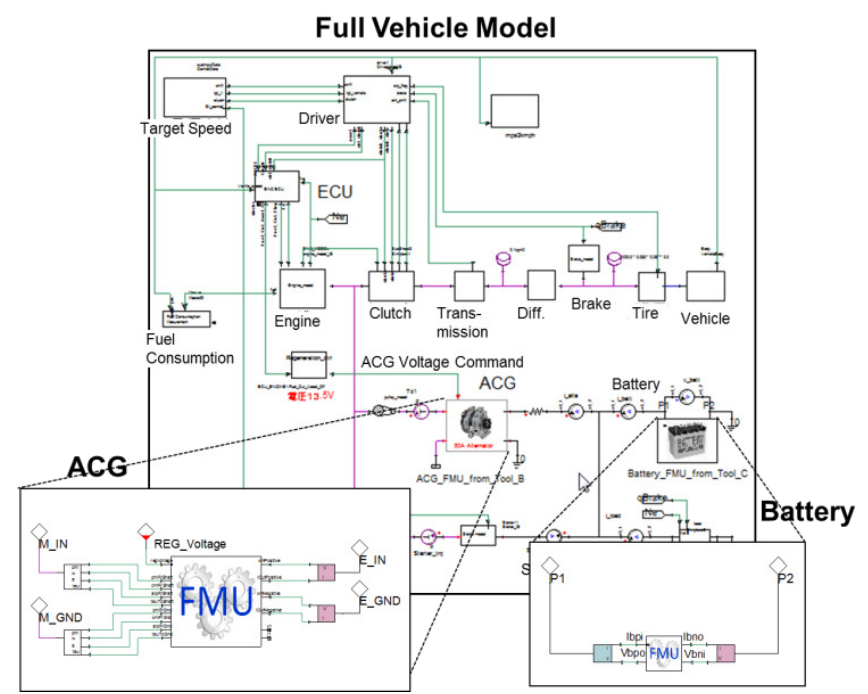

Figure 18. Full vehicle model using FMUs with adaptor models

This technique enabled us to connect sub-models developed by different tools (for example, Modelica tool and VHDL-AMS tool), though it is necessary to make adaptor models in each tool. Additionally we made a guideline for using this technique and published in the web page of JSAE (JSAE Committee, 2014). (Currently only available in Japanese.)

As future works, we plan to do following tasks.

- Extend this method also for FMI for CoSimulation.
- Enhance activity for model development and exchange between automotive industries using FMI.

- Push tool vendors to support the newest version of FMI.

- Request to make better specification of FMI for actual usage.

As for the last activity, there is high expectation to the activity by FMI Working Group of Modelic Association to realize that future FMI will support automatic decision of causality of signals (i.e. the definition of input and output). Currently user should decide the causality of signals of FMU so that there is no conflict when combining multiple models, but this task is very troublesome. Additionally it is desired that as much as possible tools will support automatic handling of algebraic loops generated by connecting multiple FMUs by utilizing above capability.

\section{References}

JSAE Committee on Research of Model Development and Circulation Methods Based on Global Standardized Description, Guideline of Model Connection using FMI in Acausal Modeling Tools, 2014 (Available online as http://www.jsae.or.jp/tops/topics/1241/1241-1A.pdf).

T. Sekisue, K. Tsuji, M. Ogawa, T. Fukada, K. Tanimoto, S. Hikida, M. Ueda, T. Kato, Alternator model for full vehicle simulation, Proc. JSAE annual conference 2013 Spring, No.407-20135490, 2013 (in Japanese).

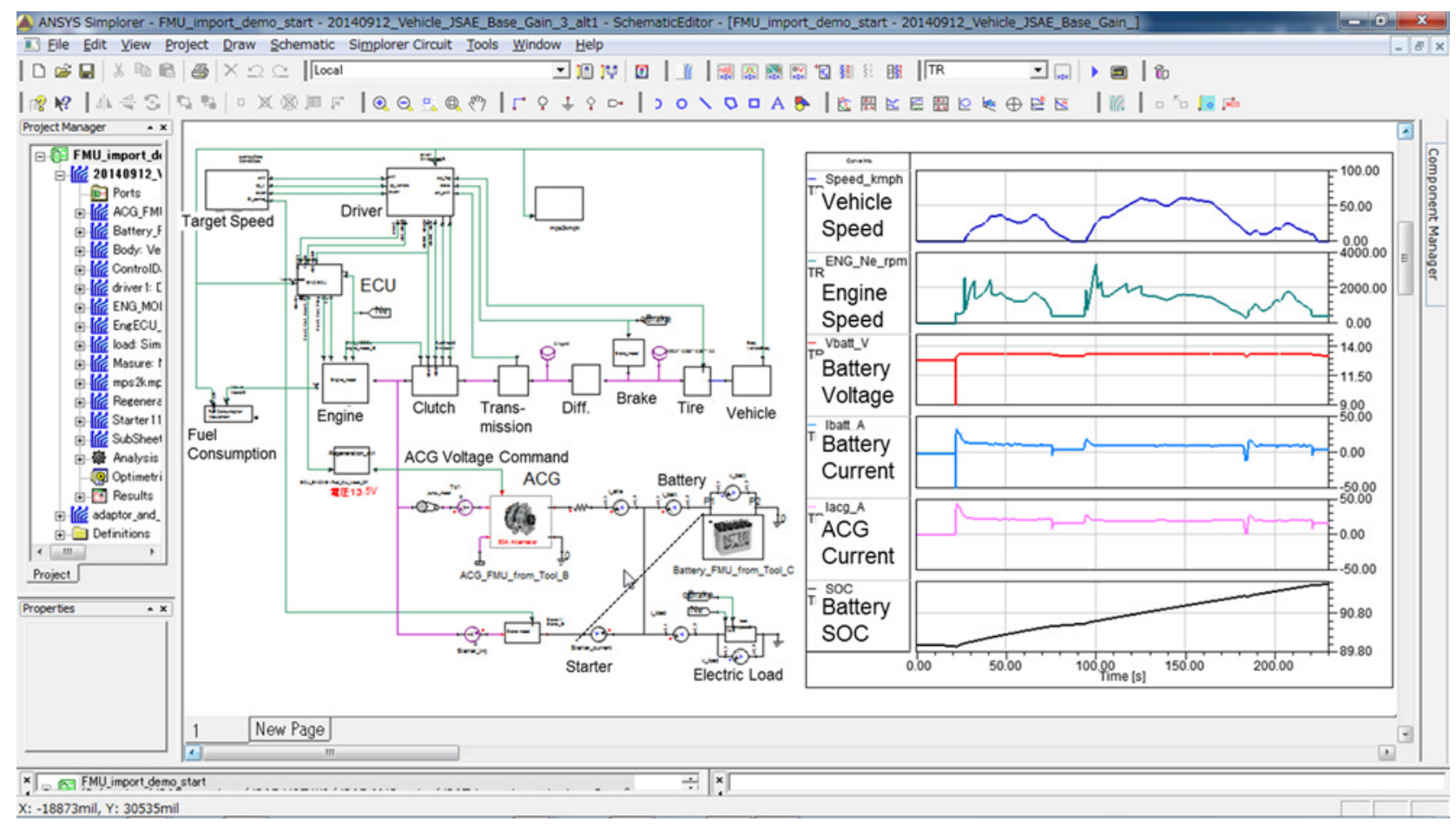

Figure 19. Simulation result of the full vehicle model 